\title{
Les récents développements dans le domaine de l'ingéniérie française en matière d'énergie hydroélectrique
}

\author{
Recent developments in the field \\ of french engineering relating \\ to hydro-electric generation
}

\author{
H. de Maublanc
}

Délégué Général de l'UNIPEDE

Pratiquement centenaire, l'hydroélectricité, dispose encore d'un avenir assuré ou prometteur ; les estimations du tableau I, fournies par la Conférence Mondiale de l'Energie d'Istanbul en 1977, en témoignent.

En France, depuis la fin du $19^{\mathrm{e}}$ siècle l'hydroélectricité n'a cessé de se développer ; déjà très sollicité depuis 30 ans, en particulier par l'important effort d'investissement dans les années 1950, l'équipement se poursuit : les stations de pompage devraient représenter en l'an 2000 entre 5 et $7 \%$ de la puissance totale du parc de production, ce qui entraînera l'engagement, tous les quatre à cinq ans, d'un aménagement de pompage de plusieurs centaines de MW, et de quelques aménagements gravitaires qui pourraient au total représenter un productible supplémentaire de 3 à 5 TWh.

Face à ce programme national et aux perspectives que peuvent représenter nos exportations, les moyens dont dispose notre pays dans les domaines de l'ingénierie sont tout à fait exceptionnels; ce sont :
- les Maîtres d'ouvrage et Maître d'œuvre (E.D.F., C.N.R. etc.) avec leurs bureaux d'études et leurs hommes de terrain;

- les Sociétés d'ingéniérie dans les domaines du génie civil et de l'électromécanique ;

- les Entreprises de travaux publics ;

- les fournisseurs d'équipement électrique, d'automatisme et de régulation;

- les constructeurs d'alternateurs, de turbines hydrauliques, de pompes-turbines, de vannes, de conduites forcées, etc. ;

- les laboratoires et plateformes d'essais.

\section{Le génie civil}

Dans le domaine du génie civil, la construction des barrages occupe une place de choix et la France une position de tout premier ordre sur la scène mondiale.

\begin{tabular}{|c|c|c|c|c|}
\hline \multicolumn{5}{|c|}{ Tableau I } \\
\hline \multirow[t]{2}{*}{ Groupes de pays } & \multicolumn{4}{|c|}{$\begin{array}{l}\text { Moyenne des investissements hydroélectriques annuels } \\
\text { entre les années indiquées (en milliards de dollars US 1976) }\end{array}$} \\
\hline & $1976-1985$ & $1985-2000$ & $2000-2020$ & $1976-2020$ \\
\hline Pays de l'OCDE & 5,05 & 3,70 & 7,71 & 5,80 \\
\hline $\begin{array}{l}\text { Pays à planification } \\
\text { économique centrale }\end{array}$ & 3,39 & 7,10 & 18,45 & 11,50 \\
\hline $\begin{array}{l}\text { Pays en voie de } \\
\text { développement }\end{array}$ & 5,64 & 10,64 & 23,17 & 15,31 \\
\hline Total mondial & 14,08 & 21,44 & 49,33 & 32,61 \\
\hline
\end{tabular}




\section{Barrages}

\section{Conception}

\section{Barrages en béton (fig. 1)}

Barrages dans les vallées étroites : il faut souligner les efforts faits par les projeteurs pour la meilleure utilisation du peu de place disponible. L'imbrication des divers éléments (barrage, évacuateur de crues, usine) est un facteur, sinon de simplification, du moins d'économie, surtout si l'on manque de place. Tels sont les barrages-usines-déversoirs de l'Aigle, Bort et Chastang, sur la Dordogne, Monteynard, etc.

Les évacuateurs en saut de ski, inventés par André Coyne, ont été pour beaucoup dans la réussite de ces ouvrages complexes (meilleure sécurité vis-à-vis des problèmes créés par l'érosion régressive, solution légère et élégante pour lâcher l'eau par dessus les usines dans l'axe des vallées). Ils sont aujourd'hui mondialement reconnus comme une solution très avantageuse.

Barrages dans les vallées larges: il faut mentionner :

- La conquête des vallées larges par le barrage-voûte : conquête longue et prudente, le maximum du rapport "longueur/hauteur" augmentant progressivement à partir de la valeur 5 (années 30) pour atteindre 12 (en 1972) à Hendrick Verwoerd (Afrique du Sud, lon-

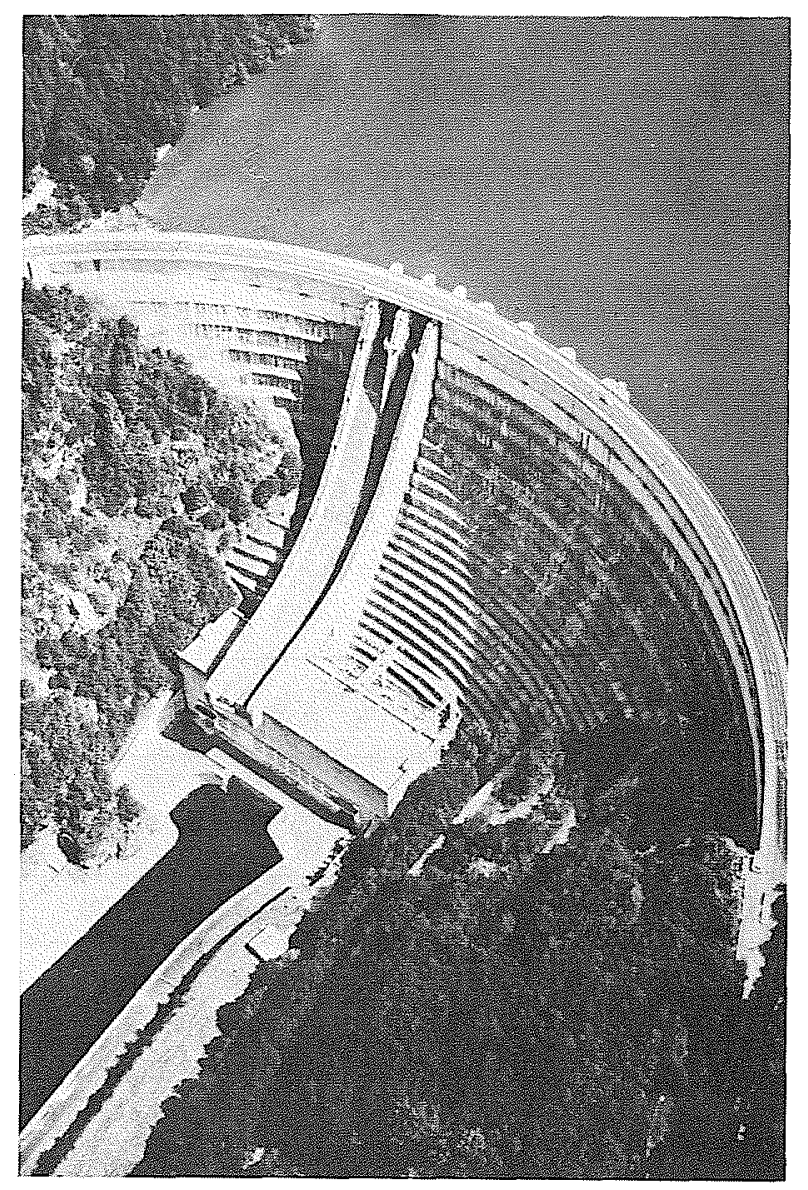

gueur $=1100 \mathrm{~m}$, hauteur $=90 \mathrm{~m}$ ). Elle s'est développée grâce au perfectionnement des méthodes de calcul (évaluation précise des contraintes) et grâce à certains ouvrages expérimentaux (exemple : voûtebatardeau de Kariba-Zimbabwe, $1 / \mathrm{h}=16$ ).

- Le progrès des voûtes multiples : ce type de barrage s'adapte parfaitement aux larges vallées à fondation rocheuse. Mais, jusque vers les années 50 , la construction des voûtes mutiples, de faible portée entre contreforts, était si difficile que l'économie procurée n'apparaissait pas dans le coût final : elle n'est apparue qu'avec l'adoption des grandes portées. Les voûtes multiples ont également conquis progressivement les hauteurs. Exemples : Granval (1959); Daniel Johnson (Canada, hauteur $214 \mathrm{~m}$ ).

Le barrage de Roselend : Ouvrage composite remarquable, constitué d'un barrage à contreforts surélevant un éperon rocheux et prenant appui, au droit de la traversée de la gorge, sur une voûte tronquée dans sa partie supérieure, sans culée, d'une hauteur maximale de $150 \mathrm{~m}$. On apprécie mieux la parfaite maîtrise des ingénieurs français quand on tient compte du fait qu'à l'époque (années 50) il n'existait pas de moyens de calcul d'une telle forme (fig. 2).

Evacuateurs de crues de grandes sections, sous fortes charges : Une autre innovation française, qui se généralise mondialement, est l'évacuation en charge à travers

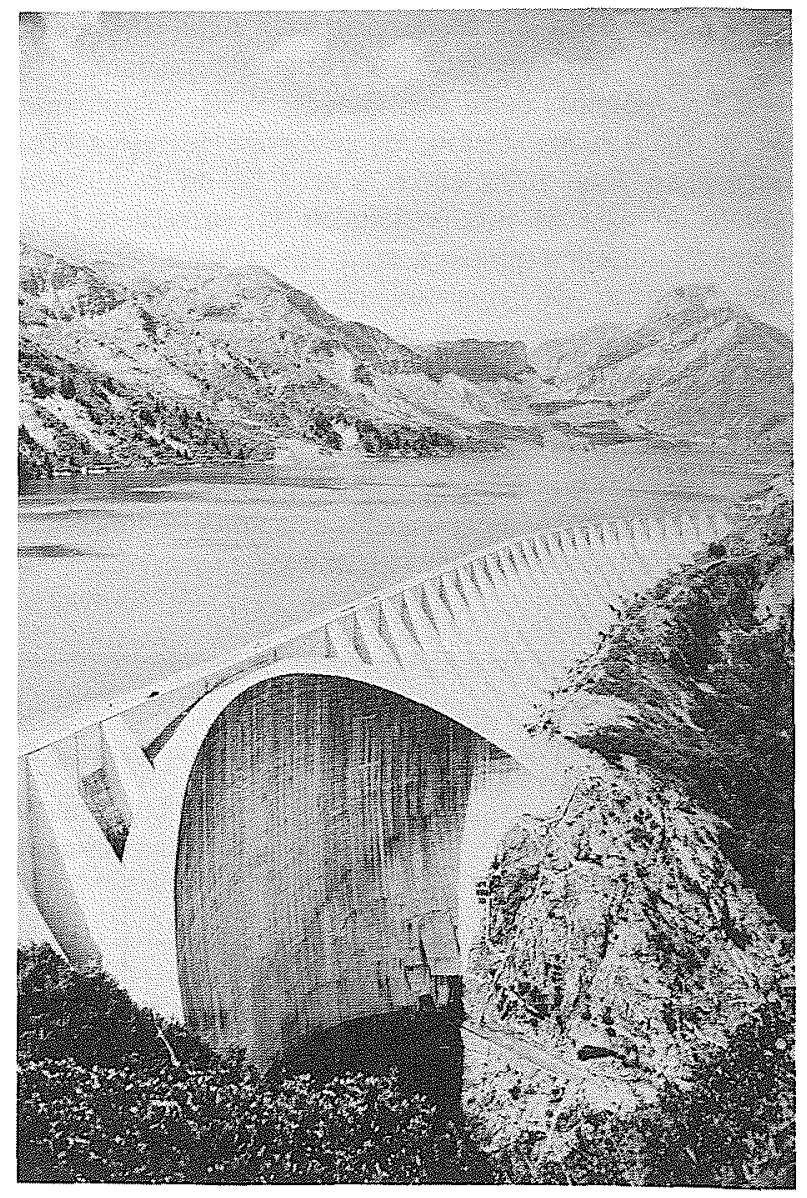

Figure 1 - Barrage-usine de Bort-les-Orgues.

Figure 2 - Barrage de Roselend. 
le barrage. Il a reçu sa consécration à Kariba, avec $9500 \mathrm{~m}^{3} / \mathrm{s}$ sous $35 \mathrm{~m}$ de charge, puis à Cabora-Bassa au Mozambique, avec $13000 \mathrm{~m}^{3} / \mathrm{s}$ sous $80 \mathrm{~m}$ de charge. A signaler, en France, les deux évacuateurs de fond du barrage-voûte de Sainte-Croix (2 pertuis de $4,50 \mathrm{~m} \times 4 \mathrm{~m}$, évacuant $1100 \mathrm{~m}^{3} / \mathrm{s}$ sous $72 \mathrm{~m}$ de charge).

\section{Barrages en remblai}

Le nombre de ces barrages mis en service en France, au cours de cette dernière décennie, dépasse celui des barrages en béton; les sites sont en général des vallées larges avec fondations non rocheuses et beaucoup, de hauteur modeste, sont destinés à l'alimentation en eau potable, industrielle ou agricole. A signaler cependant les grands barrages d'EDF tels que :

- Mont-Cenis (1968) ; hauteur : $120 \mathrm{~m}$, volume de matériaux : 14,8 millions de $\mathrm{m}^{3}$ (fig. 3).

- Grand' Maison : hauteur : $160 \mathrm{~m}, 12,5$ millions de $\mathrm{m}^{3}$.

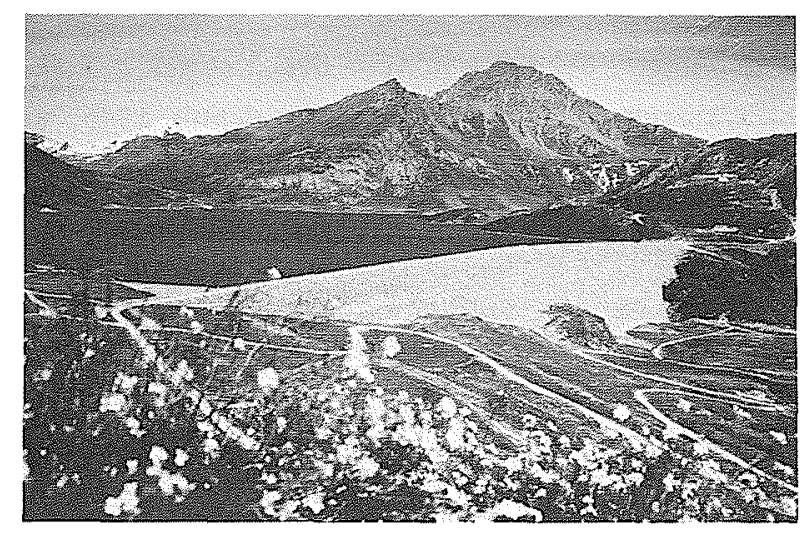

Figure 3 - Barrage du Mont-Cenis.

Les méthodes de conception et de construction, sont éprouvées ; seuls quelques aspects particuliers méritent cependant d'être signalés, ce sont :

Concentration des ouvrages : un exemple remarquable est donné par le barrage de Djatiluhur (Indonésie); toutes les fonctions hydrauliques sont groupées dans le même ouvrage qui est constitué par une énorme tour, de $90 \mathrm{~m}$ de diamètre, disposée à l'amont du barrage (fig. 4).

Organes d'étanchéité : Signalons quelques solutions nouvelles :

- masque amont en béton de brai-vinyle des digues de Montézic (hauteur maximale : $57 \mathrm{~m}$ );

- masque par membrane en chlorure de polyvinyle (2 $\mathrm{mm}$ d'épaisseur) adopté pour le barrage de Codole (hauteur $28 \mathrm{~m}$ );

- paroi interne en béton plastique (Le Drennec, hauteur $25 \mathrm{~m})$;

- noyau interne réalisé par injection des enrochements de la digue-batardeau de la centrale nucléaire de Flamanville.
Utilisation de géotextiles : à signaler les développements récents de l'utilisation, comme filtres et drains, des géotextiles constitués de fils synthétiques tissés ou non-tissés.

\section{Etude et traitement des fondations}

Etudes des fondations : depuis de nombreuses années, des équipes de géologues et de géotechniciens travaillent, soit au sein d'établissements comme E.D.F., soit au sein de bureaux d'études spécialisés. De nombreux pays étrangers font appel à leur compétence.

La mécanique des roches appliquée aux fondations de barrages : elle a connu, au cours de ces dernières années, un essor considérable dans notre pays, qui a acquis, dans ce domaine, une réputation mondiale, par les progrès réalisés dans l'interprétation du comportement des milieux fissurés, dans l'hydraulique des percolations en fissure et dans les méthodes de calcul de la stabilité des massifs rocheux. Ces développements trouvent de nombreuses applications dans l'étude des fondations rocheuses des barrages.

Traitement des fondations : Citons la première mondiale que constitua l'injection des alluvions de la Durance sous le barrage de Serre-Ponçon, sur $100 \mathrm{~m}$ de profondeur. Les injecteurs français sont devenus à cette occasion et sont restés, depuis, des "leaders" dans la maîtrise des techniques d'injection par l'invention de coulis nouveaux et de dispositifs originaux (comme les tubes à manchettes) devenus maintenant classiques.

Signalons aussi l'utilisation, de plus en plus fréquente, de la paroi moulée en béton plastique pour la réalisation de coupures étanches dans les fondations alluviales; la Société Soletanche a mis au point l'hydrofraise, qui descend dans des terrains relativement durs et jusqu'à des profondeurs de l'ordre de $100 \mathrm{~m}$.

\section{Méthodes de calcul}

Les ingénieurs français ont participé aux progrès des méthodes de calcul des barrages dans deux domaines distincts.

Les méthodes informatiques : l'informatique a d'abord été employée pour le calcul des barrages-voûtes par la Trial Load Method, mise au point à l'origine
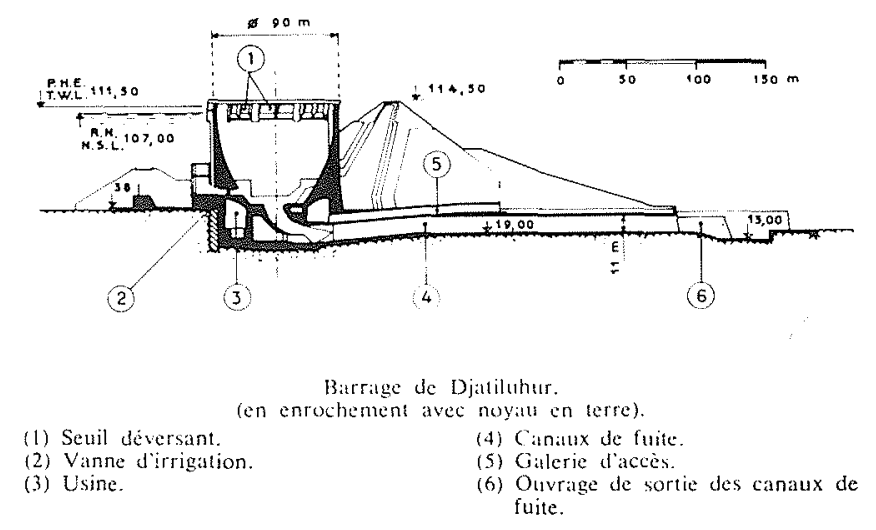

Figure 4 - Barrage de Djatiluhur. 
par les Américains. L'emploi de l'ordinateur a permis, ensuite, de supprimer les tâtonnements qui étaient liés à la méthode même ("trial"). Le meilleur programme existant actuellement est français (Coyne et Bellier). D'autre part, les développements de la Méthode des éléments finis ont été suivis et exploités en France (notamment avec la collaboration d'EDF et de l'Ecole Centrale des Arts et Manufactures) pour résoudre les problèmes spécifiques des projets de barrage, tant en béton qu'en remblais. La puissance de l'outil est telle qu'on peut aujourd'hui traiter des problèmes non linéaires et à trois dimensions.

Les méthodes semi-probabilistes : sont maintenant réglementairement introduites en France pour le calcul des structures. Dans le cas des fondations et des remblais, il faut recourir à des méthodes basées sur l'analyse paramétrique, où toute l'attention du calculateur se porte sur le poids relatif des paramètres d'un problème. La notion du coefficient de sécurité, nombre unique prétendant mesurer la sécurité, doit être abandonnée. Cette démarche, patiemment suivie en France pour l'étude des appuis rocheux de barrage, commence à être admise généralement.

\section{Construction}

Deux procédés de construction méritent d'être signalés. Il s'agit :

Construction de barrages mobiles, sur terre ferme, en dehors du lit mineur

Dans les derniers aménagements d'EDF sur le Rhin (Rhinau et chute aval) et ceux de la CNR sur le Rhône, les barrages mobiles fondés sur une épaisse couche d'alluvions sablo-graveleuses ont été construits sur terre ferme, en dehors du lit mineur, à l'abri de diguettes insubmersibles, moyennant un rabattement de nappe.

Construction d'ouvrages, dans l'eau, sans mise à sec du sol de fondaton

Une conception originale vient de trouver une application à Denouval, sur la Seine. L'ouvrage (75 $\mathrm{m}$ de largeur entre culées ; mis en service en 1980) constitue une première mondiale.

Ce nouveau type de bouchure, conçu et mis au point par le Professeur J. Aubert, est dénommé "clapets à vérins aval". La nouveauté réside dans le dispositif de manœuvre hydraulique : c'est un vérin à double effet, logé dans le radier à l'aval du clapet, qui assure son relevage.

Le procédé de construction consiste à coiffer par un couvercle, l'espace compris entre deux rideaux de palplanches parallèles battus en travers du lit et à remplir la boîte ainsi formée par du béton à la pompe. Le radier métallique, auquel est incorporée la bouchure mobile, peut être construit sur un chantier naval, transporté par flottaison et échoué sur le site.

Par rapport à la construction classique, une économie de $30 \%$ a été réalisée à Denouval par ce procédé.

\section{Inspection et surveillance des barrages}

Le contrôle de la sécuirté des barrages en France, peut sans doute servir de modèle tant par ses dispositions administratives et réglementaires que par l'accent mis sur l'importance de la qualité technique.

\section{Réglementation française en matière de barrages}

Le Comité Technique Permanent des Barrages (organisme interministériel) est obligatoirement consulté sur les avant-projets et projets d'exécution des barrages dont la hauteur est au moins égale à $20 \mathrm{~m}$.

La circulaire interministérielle du 14 Avril 1970 traite des mesures à prendre pour assurer le contrôle et la surveillance des barrages, au cours de leur première mise en eau et pendant toute leur durée de vie.

Enfin, le décret du 16 Mai 1968 a rendu obligatoire la mise en place de plans d'alerte pour tous les barrages de plus de $20 \mathrm{~m}$ de hauteur et de capacité de retenue au moins égale à $15 \mathrm{hm}^{3}$. De nombreux pays étrangers consultent la France sur les problèmes de calcul de l'onde de submersion et les dispositifs techniques d'alerte aux populations à l'aval des barrages.

\section{Organisation de l'auscultation des barrages à E.D.F}

L'auscultation des barrages est, depuis 1946, une préoccupation majeure d'EDF : le suivi des mesures d'auscultation porte actuellement sur quelque $150 \mathrm{bar}$ rages. Cette organisation sert également de modèle à l'étranger.

Il faut signaler l'utilisation récente, pour les visites décennales, d'un petit sous-marin permettant d'éviter la vidange de la retenue.

\section{Prises d'eau de haute montagne à chasses auto- matiques}

Le problème des prises de haute montagne, en général nombreuses et souvent situées dans des endroits d'accès difficile, a pu être résolu grâce à la mise au point par EDF des prises d'eau à chasses automatiques; des ouvrages de ce type ont été adoptés par un certain nombre de pays étrangers. Il y a lieu de signaler également l'originalité des prises sous-glaciaires (prises de l'Argentière et du Tour dans l'aménagement d'Emosson et prise sous la Mer de Glace dans l'aménagement des Bois).

\section{Ouvrages souterrains}

Un grand nombre des usines hydroélectriques classiques d'EDF construites en montagne depuis deux décennies, ont été installées en souterrain. Ce choix s'explique pour de multiples raisons : exiguité du site, qualité des terrains, économie sur le circuit hydraulique, enfoncement des groupes de pompage, protection de l'environnement, etc.

\section{Perforation des galeries au tunnelier}

L'utilisation des tunneliers continue à se développer dans notre pays pour la perforation des galeries et puits 
inclinés d'adduction des aménagements hydroélectriques.

Le tunnelier présente l'avantage de minimiser les hors-profils, les soutènements et les revêtements. De plus, la surface du rocher brut de dérochement peut, sous certaines précautions, présenter un coefficient d'écoulement suffisant pour que soit économisé le revêtement, souvent destiné à la seule réduction des pertes de charges. Enfin, le tunnelier améliore les conditions de travail et la sécurité du personnel d'exécution.

\section{Exemples d'utilisation du tunnelier}

- la galerie d'Echaillon (1972-1973) : diamètre d'excavation $5,80 \mathrm{~m}$, longueur $4700 \mathrm{~m}$;

- la galerie de Bramefarine (Arc-Isère, 1976-1977) : diamètre d'excavation $8,10 \mathrm{~m}$, longueur $3800 \mathrm{~m}$; - les 4 puits inclinés de Grand'Maison (1979-80 et 1980-81) : diamètre d'excavation $3,60 \mathrm{~m}$, pente $56 \%$; $1480 \mathrm{~m}$ de longueur chacun ;

- le puits incliné de Super-Bissorte (1980-81) : diamètre d'excavation $3,60 \mathrm{~m}$, pente 43 à $51 \%$, longueur 2800 .

\section{Conception des usines souterraines}

Deux tendances se font jour :

- l'une consistant à loger l'essentiel du matériel dans une même excavation;

- l'autre cherchant à réduire le plus possible la portée de l'excavation principale abritant les groupes en installant le matériel annexe dans des chambres indépendantes.

\section{Exemples}

- A l'usine souterraine de Montézic (en service prochainement) il y a une seule excavation (longueur $145 \mathrm{~m}$, largeur $25 \mathrm{~m}$ et hauteur $41 \mathrm{~m}$ ) abritant les 4 groupes, les vannes amont, les vannes aval et les transformateurs.

- A l'usine souterraine de l'Eau d'Olle (aménagement de Grand'Maison, en cours de réalisation), il y a trois excavations : la salle des machines (longueur $161 \mathrm{~m}$, largeur $15 \mathrm{~m}$ et hauteur $39 \mathrm{~m}$ ) abritant les 8 groupes, la chambre des robinets amont et la chambre des batardeaux aval.

\section{Utilisation du prédécoupage et du béton projeté pour les grandes cavernes}

Parmi les progrès récents des techniques de perforation et de soutènement, il convient de retenir pour les grandes cavernes :

- l'intérêt du prédécoupage qui limite les ébranlements et la détérioration du rocher et conduit à d'importantes économies de soutènement et de revêtement;

- le développement de l'emploi du béton projeté comme soutènement d'excavations souterraines; il est mis en place immédiatement après le déroctage. Dans le cas de roches dures, peu fracturées, le béton projeté peut assurer seul un rôle de soutènement; dans le cas de roches fracturées, il est associé à un boulonnage jouant le rôle primordial.

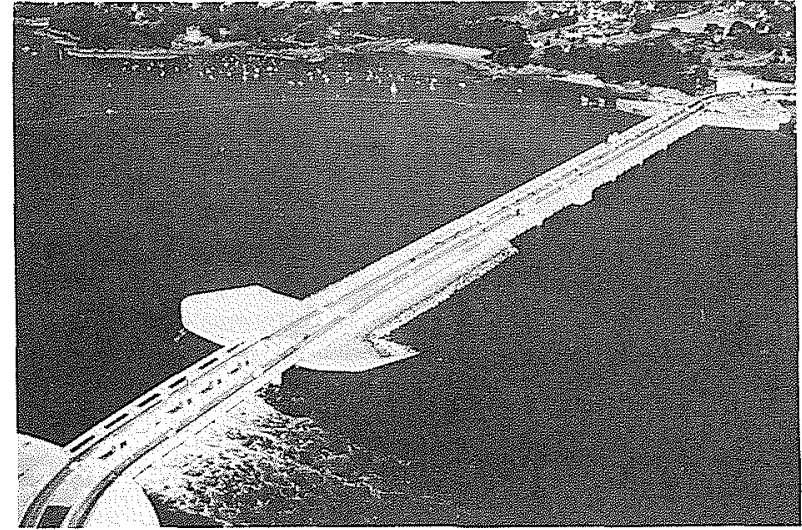

Figure 5 - Turbine marémotrice de la Rance.

L'usine marémotrice de la Rance et les perspectives offertes pa l'énergie marémotrice

Depuis presque le début du siècle, les ingénieurs français se sont intéressés à l'énergie marémotrice pour la production d'électricité. L'estuaire de la Rance, où l'amplitude de la marée atteint $13,50 \mathrm{~m}$, a été considéré par EDF comme le meilleur site pour l'implantation d'une usine marémotrice de taille industrielle et l'aménagement (1961-1967) constitue une première mondiale (fig. 5).

La situation privilégiée des côtes de la Manche au point de vue amplitude des marées et l'expérience de l'usine de La Rance, devaient tout d'abord profiter à notre pays. La situation énergétique actuelle et l'intérêt que présentent les énergie nationales et renouvelables ont amené EDF à rouvrir le dossier de l'énergie marémotrice. Les études ont été reprises en vue tout d'abord de dresser l'inventaire des sites envisageables le long des côtes de la Manche (potentiel équipable de l'ordre de $40 \mathrm{TWh} / \mathrm{an}$ ). Puis, il est apparu utile d'engager des études de faisabilité sur un site assez modeste ( 5 à $6 \mathrm{TWh}$ ), mais de taille suffisante pour, d'une part, occuper une place intéressante dans le parc de produc. tion et, d'autre part, constituer un support d'études valables en regard des problèmes posés par un aménagement marémoteur dans les domaines technique, économique et environnementaliste. Ces études devraient permettre de disposer des éléments d'appréciation nécessaire à toute prise de décisions.

\section{Le matériel électro-mécanique}

\section{Aspects généraux}

Deux faits ont marqué l'hydroélectricité récente en France

- le ralentissement de l'hydraulique gravitaire classique (dû à la raréfaction progressive des sites disponibles et aux contraintes d'environnement),

- l'essor des stations de transfert d'énergie par pompage - STEP - (lié au développement des centrales nucléaires). 
Augmentation des caractéristiques des groupes (puissance unitaire, hauteur de chute, vitesse de rotation) tants :

Elle pose aux constructeurs des problèmes impor- grandes dimensions des pièces et fortes pressions qui entraînent des études hydrauliques et mécaniques très poussées (étude des fréquences propres, des phénomènes transitoires, cavitation, statique et dynamique des structures paliers et pivots, joints tournants, etc.);

- le mode de réfrigération et le système de fixation des pôles, dans le domaine des alternateurs.

\section{Mode d'exploitation des groupes - Chaines d'auto- matisme}

L'exploitation des groupes importants se fait presque toujours maintenant par télécommande depuis un dispatching ou un Poste de Commande Hydraulique (PCH) et, en secours, à l'aide de programmateurs locaux.

Dans le cas des STEP où les chaînes d'automatisme sont les plus nombreuses et les plus complexes et où le nombre journalier de changements d'état des groupes est également le plus élevé, le choix est orienté vers des équipements à base d'automates à logique programmable (le Controbloc à Montézic et le $\mathrm{H} 20$ au Pouget et au Truel). D'autres automates plus simples sont également utilisés dans des aménagements de moindre importance.

\section{Participation des groupes aux réglages secondaires de la fréquence et de la tension}

Téléréglage : Les groupes de puissance élevée munis d'un distributeur réglable sont de plus en plus nombreux à participer au réglage secondaire de la fréquence (téléréglage) bien que celui-ci, en modifiant la puissance programmée, puisse dans certains cas changer sensiblement les conditions normales d'exploitation.

Réglage secondaire de la tension : Une quarantaine de groupes hydrauliques participent à ce réglage créé en 1979 et qui, selon les prévisions, doit concerner plus d'une centaine d'unités.

\section{Aspects particuliers}

Les principales mises en service au cours de la dernière décennie concernent :

\section{Turbines Francis}

- Sisteron (2 groupes de $120 \mathrm{MW}$, en 1974 et 1975)

- Brommat Ir (1 groupe de $240 \mathrm{MW}$, en 1975).

Les quelques groupes en cours de construction sont presque tous destinés à suréquiper des usines anciennes (au Pouget : 1 groupe de $257 \mathrm{MW}$; à l'Aigle ; 1 groupe de $133 \mathrm{MW}$, à Couesque : 1 groupe de $61 \mathrm{MW}$, etc.). A part le groupe de Brommat II qui possède une bâche spirale à double coque, aucun autre groupe ne présente de particularité notable.

\section{Turbines Pelton}

- Chatelard-Vallorcine (3 groupes ternaires munis chacun d'une Pelton de $64 \mathrm{MW}$, en 1973)

- La Combe d'Avrieux (1 groupe de $121 \mathrm{MW}$, en 1975).

Sont actuellement en cours de réalisation :

- Grand'Maison (4 groupes de $138 \mathrm{MW}$ sous $840 \mathrm{~m}$ )

- Super-Bissorte ( 1 groupe de $143 \mathrm{MW}$ sous $1114 \mathrm{~m}$ ).

\section{Turbines Kaplan}

- Salignac (2 groupes de 43 MW, en 1976).

Sont actuellement en cours de réalisation :

- Villerest (2 groupes de $30 \mathrm{MW}, \phi$ roue $3,40 \mathrm{~m}$ )

- Oz (1 groupe de $11,5 \mathrm{MW}, \varnothing$ roue $2,25 \mathrm{~m}$ ).

\section{Turbines à écoulement axial :}

L'aménagement du tronçon français du Rhin, s'est achevé en 1974 par la mise en service de l'usine de Gambsheim ( 4 groupes bulbe de $24 \mathrm{MW}$, diamètre de roue $5,60 \mathrm{~m}$ ).

Sur le Rhône en aval de Lyon, la construction des aménagements comportant des groupes bulbe qui avait commencé en 1966 par celle de Pierre Bénite (4 groupes de $20 \mathrm{MW}$ ) s'est achevée, en 1980 par celle de Vaugris (4 groupes de $18 \mathrm{MW}$ ). Sur le haut Rhône, l'aménagement de Chautagne ( 2 groupes de $45 \mathrm{MW}$ ) a été achevé en 1980, celui de Belley le sera en 1982 et celui de Bregnier-Cordon en 1984; les 2 derniers (Loyette et Sault-Brenaz) sont en cours de procédure.

Des études ont été engagées récemment pour définir l'équipement d'usines marémotrices : une nouvelle famille de groupes bulbe pourrait être définie; la réalisation de ces usines permettrait de donner un nouvel essor aux groupes à écoulement axial.

\section{Pompes-turbines}

Le choix des groupes binaires réversibles préférés aux groupes ternaires utilisés autrefois, a permis de réaliser des économies importantes sur le génie civil grâce à leur faible encombrement et à la simplification des installations.

Leur puissance unitaire et la hauteur de chute sous laquelle on les fait fonctionner, ont progressé considérablement :

Pompes-turbines monoétages : Utilisées lorsque la hauteur de chute est inférieure à $600 \mathrm{~m}$ environ, elles peuvent être munies d'un distributeur réglable comme les turbines classiques et participer comme elles aux réglages primaire et secondaire de la fréquence - Vouglans : 1 groupe de $66 \mathrm{MW}$, en 1973 ;

- Sainte-Croix : 1 groupe de 48 MW, en 1975-1977;

- Revin : 4 groupes de $168 \mathrm{MW}$, en 1975-1976

- Cheylas : 2 groupes de 209 MW, en 1980.

Parmi les réalisations en cours ou décidées :

- Montézic : 4 groupes de $217 \mathrm{MW}$, mise en service prévue pour 1982-83.

- Redenat: 3 groupes de $350 \mathrm{MW}$, mise en service prévue pour 1989. 
Pompes-turbines multiétages : Au delà de $600 \mathrm{~m}$ environ de hauteur de chute, la roue comporte obligatoirement plusieurs étages et il devient alors très difficile d'installer un distributeur mobile devant chacun d'eux, d'où l'impossibilité de participer au réglage continu de la fréquence et obligation de démarrer en pompe roue noyée (d'où un couple de démarrage élevé).

Quatre machines de ce type, de $80 \mathrm{MW}$, installées dans l'usine de La Coche sont en service depuis 1977 ; 12 sont en construction : 8 de $156 \mathrm{MW}$ à Grand'Maison (fig. 6) (mise en service pour 1984-1985) et 4 de $158 \mathrm{MW}$ à Super-Bissorte (mise en service pour 1985 . 1986).

A l'occasion de la définition des caractéristiques des groupes de Grand'Maison, des études sur modèle réduit ont été faites pour définir la faisabilité de pompes. turbines à 2 étages munis chacun d'un distributeur réglable. En outre, un prototype est en cours d'installation à l'usine de Truel (1 groupe de $38 \mathrm{MW}$, sous $443 \mathrm{~m}$ de chute, mise en service en 1982) ; son extrapolation permettra de définir les groupes destinés à équiper des projets ultérieurs particulièrement intéressants et dont la hauteur de chute est comprise entre 750 et $1050 \mathrm{~m}$ (Le Clou, Orlu, etc.)

\section{Alternateur-moteurs}

Le développement des alternateurs-moteurs a suivi naturellement celui des pompes-turbines. Des pro-

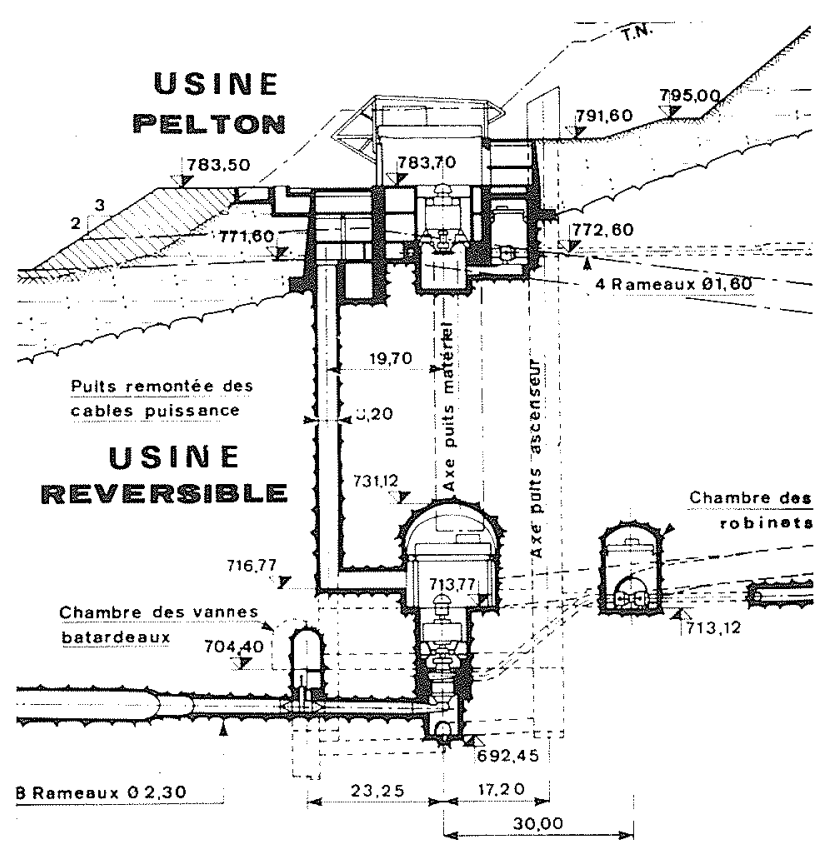

Figure 6 - Usine de Grand'Maison. blèmes liés à des procédés de démarrage en pompe sont posés aux constructeurs électriciens :

- démarrages par machine électrique auxiliaire fixée sur l'arbre du groupe principal (moteur Poney).

- démarrages asynchrones directs sur le réseau à pleine tension (cas de Vouglans, Ste-Croix, groupe $\mathrm{n}^{\circ} 4$ de Revin, La Coche) ou à tension réduite (cas de Cheylas). - démarrages à fréquence variable

- en dos à dos à l'aide d'un groupe voisin (second procédé utilisé à Vouglans et Ste-Croix - seul procédé prévu à Grand'Maison, à Super-Bissorte et au Truel)

- à l'aide d'un convertisseur statique de fréquence (cas de Montezic et Redenat).

Les démarrages asynchrones qui sont à la fois plus économiques et plus rapides que tous les autres procédés sont les plus intéressants pour le maître d'ouvrage. Mais ils provoquent sur le réseau des variations de tension (limitées en général à $5 \%$ ) et obligent les constructeurs des machines électriques à étudier avec soin et à renforcer certains éléments de leur fourniture.

Il semble que l'emploi des convertisseurs statiques de fréquence se développera rapidement surtout si le prix de ces appareils permet d'en installer un par groupe.

\section{Conduites forcées}

Modes d'installation : Pour les STEP, elles sont en général installées en galerie et bloquées au rocher; la participation de celui-ci à la résistance à la pression intérieure est prise en compte dans les calculs, ce qui permet dans certains cas de réduire l'épaisseur des viroles.

Caractéristiques : Les quelques indications ci-après permettent de se rendre compte des progrès réalisés : à Villarodin $\quad \mathrm{PD}^{2}=8,5 \times 10^{6}\left(\mathrm{bar}-\mathrm{cm}^{2}\right) ; \mathrm{V}=7,5 \mathrm{~m} / \mathrm{s}$ à Grand'Maison $\mathrm{PD}^{2}=9,9 \times 10^{6}\left(\mathrm{bar}-\mathrm{cm}^{2}\right) ; \mathrm{V}=10,40 \mathrm{~m} / \mathrm{s}$ à Super-Bissorte $\mathbf{P D}^{2}=13,3 \times 10^{6}\left(\right.$ bar- $\left.\mathrm{cm}^{2}\right) ; \mathrm{V}=9,8 \mathrm{~m} / \mathrm{s}$.

Nuances des aciers utilisés : La tendance est d'utiliser de plus en plus fréquemment des aciers à haute limite d'élasticité pour réduire les difficultés inhérentes à l'épaisseur des tôles à souder.

\section{Conclusion}

Tous ces aspects originaux et les plus marquants de l'ingéniérie française en matière d'énergie hydroélectrique, ainsi que les progrès réalisés dans son développement au cours de ces deux dernières décennies, prouvent que l'hydroélectricité française se porte bien ; et que les moyens, dont elle dispose dans les domaines de l'ingéniérie, doivent permettre de répondre tout à fait convenablement à des demandes étrangères et contribuer ainsi à développer nos exportations. 


\section{Discussion}

Président : M. M. COUPIN

M. le Président. - Je crois qu'on peut consacrer quelques minutes de discussion à cette première partie de votre exposé, avant de passer à la deuxième. Je n'ai pas besoin d'insister sur la qualité et l'intérêt de cette première partie.

Qui souhaite ouvrir le feu?

$M$. GOUBET. - J'aurais tendance à rapprocher les deux exposés, celui de M. COTILlON et celur de M. de MAUBLANC.

M. COTILLON nous a dit qu'au plan mondial on avait encore quatre buts sur cinq à marquer en matière hydroélectrique alors qu'en France on en était à jouer les prolongations. Maintenant $M$. de MAUBLANC nous informe que nous disposons d'un potentiel considerable en matière d'ingénierie, potentiel constitué à partir des réalisations françaises. La France a toujours travaillé à l'étranger dans le domaine hydroélectrique, mais désormais elle va devoir s'y consacrer presque en totalité.

N'y a-t-il pas de risques pour cette ingénierie dans ce changement d'activité? Ne craignez-vous pas qu'il y ait quelques plumes à perdre éventuellement au cours de cette mutation?

M. H. de MAUBLANC. - Bien sûr; mais j'espère quand même qu'on ne se trouvera pas complètement déplumé ; c'est un problème qui nous préoccupe, ce n'est pas douteux.

Je pense que c'est en liaison avec des bureaux d'étude extérieurs aux entreprises, maîtres d'ouvrage, comme avec les constructeurs, qu'il nous faut désormais travailler pour faire connaître davantage encore nos réalisations et les moyens dont nous disposons, et pour éviter, comme vous le soulignez, cette désaffection progressive. Evidemment ce n'est pas toujours facile.

Nous avons en ce domaine pris un certain nombre de contacts, et nous continuerons à en prendre, en liaison d'ailleurs avec les Pouvoirs Publics et les organismes d'exportation. On peut organiser des tournées de conférences pour mieux faire connaître nos réalisations, ou multiplier les réunions comme celles dont la Société Hydrotechnique de France a pris l'initiative. M. CAZENAVE a rappelé que nous avions ici des amis venus de pays étrangers : je m'en félicite largement

En dehors de cela je n'ai effectivement pas de réponse très pertinente ou très claire à vous proposer, mais soyez assuré que c'est un problème dont nous mesurons toute l'ampleur.

$M$. le Président. - Effectivement, c'est un problème important. Mais je crois que l'ingénierie française a montré sa capacité dans le passé en France et dans le monde.

La France a eu l'honneur d'avoir quelques grands ingénieurs qui ont su faire progresser les technologies et $M$. de MAUBLANC a bien insisté sur l'importance de l'innovation; l'ingénierie française a une renommée certaine en ce domaine et je ne vois pas pourquoi elle ne continuerait pas à entretenir simultanément des relations suivies avec l'extérieur et à conserver la place qui lui revient tout naturellement en faisant preuve de sa capacité d'innovation; mais il est évident qu'il est plus facile de travailler sur son propre terrain. L'ingénierie fran- çaise devra donc fournir un effort considérable et je ne doute pas qu'elle sera sensible à l'importance de l'enjeu.

Je pense que l'on pourrait arrêter là les questions, et passer à la seconde partie de votre exposé, quitte à revenir sur la premoère partie de ce sujet en fin de séance.

Après la deuxième partie de l'exposé en M. de MAUBLANC, la discussion reprend.

M. le Président. - Il nous reste quelques minutes pour répondre aux questions de la salle sur cette deuxième partie de l'exposé de M. de MAUBLANC.

M. RANGER. - Vous avez évoqué les recherches concernant une nouvelle génération de groupes bulbes. Pourriez-vous nous indiquer en quelques mots dans quelle direction s'orientent ces recherches?

M. de MAUBLANC. - M. LESCURE qui suit attentivement ce genre de problème au sein d'EDF va vous répondre.

M. LESCURE. - Les groupes bulbes de rivière ne fonctionnent qu'en turbine. Les groupes bulbes de la Rance fonctionnent en turbine dans les deux sens et en pompe dans les deux sens également (au total quatre types de fonctionnement)

Les nouveaux groupes marémoteurs ne fonctionneront probablement qu'en turbine directe et en pompe directe (au total deux types de fonctionnement). Le tracé des pales sera donc entièrement différent des tracés actuels. Par ailleurs il n'est pas exclu que les groupes soient équipés d'un multiplicateur de vitesse.

M. AMBLARD (Neyrpic). - Pour compléter ce qui vient de dire M. LESCURE, il faut préciser que les groupes marémoteurs envisagés actuellement pour l'équipement des sites du Cotentin sont prévus pour les deux modes de fonctionnement suivants : - en turbine directe : le flux traverse le bulbe selon le cheminement : avant-distributeur, distributeur, roue, aspirateur; - en pompe directe : le flux traverse le bulbe selon litinétaire ci-après : aspirateur, roue, distributeur, avant-distributeur, soit avec un sens de rotation inverse du précédent.

C'est une turbine-pompe classique comme pour les stations de transfert d'énergie, alors que les groupes de la Rance ont des modes de fonctionnement supplémentaires qui sont :

- en turbine inversée : le flux traverse le bulbe selon le parcours : aspirateur, roue, distributeur, avant-distributeur (même sens de rotation que la pompe directe) ;

- en pompe inversée : le flux traverse le bulbe selon l'itinéraire : avant-distributeur, distributeur, roue, aspirateur (même sens de rotation que la turbine directe).

Le fonctionnement des groupes projetés pour les sites du Cotentin, étant limité à la turbine directe et à la pompe directe, devrait permettre un tracé des profils hydrauliques avec de meilleures performances pour ces deux modes que ceux correspondant aux quatre modes de fonctionnement de la Rance et qui nécessitaient un compromis de forme plus difficile. 


\section{Abstract}

\section{Recent developments in the field of french engineering relating to hydro-electric generation}

The World-scale development of hydro-electric power stations continues in France too, notwithstanding the considerable pressures on resources, where substantial means have been made available for hydro-electric generation.

As regards civil engineering work, the salient developments in the techniques in France to construct dams were: design (concrete dams, "ski-jump" spillways in narrow valleys, progress in the construction of arches in large valleys, use of confined flow spillways; embakment dams: new solutions for sealing systems, and the study and treatment of the foundations), computations (use of computer and semi-probabilistic methods), construction (two procedures should be mentioned: the construction of mobile dams on dry land, outside the lower bed and construction underwater without deponding the foundations - application at Denouval) and operation (inspection and surveillance rules). The design of water intake structures fitted with automatic scours has solved the problem of intake structures at high altitudes. Tunnelling machines are already in use in France on major developments, and are being growincly used for boring galleries and tilted shafts. The salient events as regards underground stations relate to: design (single main excavation or several excavations to house units of equipment) and boring and earth retaining techniques (value of prior cutting and development of the use of batched concrete). The prospects opened up by the use of tidal energy are reexamined; the Rance plant was still the only operating project in the World fifteen years after it entered into service. Feasibility studies in the technical, economic and environmental fields are presently under way.

The most striking aspects relating to electro-mechanical equipment are chiefly linked to the enhancement of the characteristics of sets (unit power, head, rotational speed, entailing problems of size, high pressure and cooling) and their operating mode (automatic systems and remote control, secondary regulation of frequency and voltage). The number of binary sets has also grown (considerable progress in characteristics); a prototype of a two-stage pump-turbine, each stage being fitted with a regulatable distributing valve is being installed at the Truel plant. Some progress has also been made in the generator motor field (characteristics, pump-mode startup procedure) and confined flow conduits (method of installation, new steels). 